\title{
Estratégia de educação em saúde para jovens: relato de experiência em grupo de estudantes
}

Health education strategy for young people: report of experience in student group Estrategia de educación sanitaria para jóvenes: informe de experiencia en grupo de estudiantes João Pedro de Santana SILVA ${ }^{\mathbf{P}}$

Maria Amélia Pires Soares da SILVA ${ }^{1}$ Natiely Martins LEITE ${ }^{\mathbf{1}}$

Adriana Gomes MAGALHÃES ${ }^{2}$

Laiane Santos EUFRÁSIO ${ }^{2}$

${ }^{1}$ Curso de Graduação em Fisioterapia, Universidade Federal do Rio Grande do Norte - UFRN

${ }^{2}$ Professora Adjunta do Curso de Graduação Fisioterapia da Faculdade de Ciência da Saúde do Trairí - UFRN

\section{Resumo}

O trabalho tem como objetivo relatar a experiência de uma ação de educação em saúde voltada para o público jovem, participantes de um curso preparatório para o Exame Nacional do Ensino Médio. A ação foi desenvolvida no próprio curso, pelos discentes de graduação em fisioterapia, participantes de um projeto de extensão. Para isso, utilizou-se a metodologia de sala de aula invertida com abordagem teórico-prática para a ação. Antes dela acontecer, a educação permanente em saúde foi utilizada para os estudantes de graduação, como forma de adquirir conhecimento sobre o assunto a ser abordado. Os temas abordados foram prevenção do suicídio, conscientização da saúde do períneo, prevenção de doenças cardiovasculares, prevenção do câncer de intestino. Foi possível observar que a experiência promoveu um meio de empoderamento e conscientização para os jovens, uma vez que mostraram empolgação, assim como uma vivência de educação em saúde, ensino e humanização para os graduandos.

Descritores: Promoção da Saúde; Educação em Saúde; Fisioterapia.

\section{Abstract}

The work aims to report the experience of a health education action aimed at young people, participating in a preparatory course for the National High School Exam. The action was developed in the course itself, by undergraduate students in physiotherapy, participants in an extension project. For this, the inverted classroom methodology with a theoretical-practical approach to action was used. Before it happened, permanent health education was used for undergraduate students, as a way to acquire knowledge on the subject to be addressed. The topics covered were suicide prevention, perineum health awareness, cardiovascular disease prevention, bowel cancer prevention. It was possible to observe that the experience promoted a means of empowerment and awareness for young people, since they showed excitement, as well as an experience of health education, teaching and humanization for undergraduates.

Descriptors: Health Promotion; Health Education; Physical Therapy Speciality

\section{Resumen}

El trabajo tiene como objetivo relatar la experiencia de una acción de educación para la salud dirigida a jóvenes, participantes de un curso preparatorio para el Examen Nacional de Bachillerato. La acción fue desarrollada en el propio curso, por estudiantes de pregrado en fisioterapia, participantes de un proyecto de extensión. Para ello, se utilizó la metodología de aula invertida con un enfoque teórico-práctico de la acción. Antes de que sucediera, los estudiantes de pregrado utilizaban la educación permanente en salud como una forma de adquirir conocimientos sobre el tema a abordar. Los temas tratados fueron prevención del suicidio, conciencia sobre la salud perineal, prevención de enfermedades cardiovasculares, prevención del cáncer de intestino. Se pudo observar que la experiencia promovió un medio de empoderamiento y sensibilización de los jóvenes, ya que mostraron ilusión, así como una experiencia de educación en salud, docencia y humanización para los estudiantes de pregrado.

Descriptores: Promoción de la Salud; Educación en Salud; Fisioterapia.

INTRODUÇÃO

\section{O Sistema Único de Saúde (SUS) é} efeito da articulação de uma série de forças sociais e políticas em defesa da saúde como bem público, ao mesmo tempo, é a forma como o Estado brasileiro se organizou para efetivar as políticas de saúde no país. À medida que o Sistema adota uma compreensão ampliada de saúde, objetivando superar a perspectiva hegemônica, ele se compromete com a Promoção da Saúde ${ }^{1}$.

A promoção da saúde consiste em um conjunto de estratégias e formas de produzir saúde, no âmbito individual e coletivo, visando atender as necessidades sociais de saúde e a melhoria da qualidade de vida. Na Carta de Ottawa, em 1986, representantes de 35 países assumiram que as ações de promoção da saúde deveriam resultar na redução das iniquidades em saúde, o que garantiu a todos os cidadãos a oportunidade de fazer escolhas escolhas mais favoráveis à saúde e serem protagonistas no processo de produção da saúde e melhoria da qualidade de suas vidas ${ }^{2}$.

Nessa perspectiva, surge a necessidade de promover mudanças/transformações na formação de profissionais da saúde, quer no âmbito dos cursos de graduação e pósgraduação, quer no âmbito dos serviços, por meio de ações de educação permanente, o que acentuou a preocupação com as relações entre as instituições que compõem o sistema de formação de pessoal e o sistema de saúde, tema que desafia estudiosos da área, gestores e profissionais de saúde ${ }^{3}$.

Diante disso, emerge a prática de educação em saúde. Entende-se por educação a utilização de processos e técnicas pedagógicas para a socialização de conhecimentos e formação de sujeitos, tendo como base as diversas relações humanas. 
Assim, pode-se conceituar a educação em saúde como a utilização desses processos e técnicas para o compartilhamento de saberes sobre saúde que podem influenciar o cotidiano das pessoas, possibilitando a melhoria de sua qualidade de vida ${ }^{4}$.

Dentre os grandes desafios das equipes, a educação em saúde tem papel de destaque, por ser um espaço com possibilidade de fomentar reflexões, contribuir com mudança de práticas e posturas, que busquem promover a saúde e prevenir a manifestação de doenças ${ }^{5}$.

Logo, a educação em saúde apoia-se nos pressupostos da promoção da saúde, o qual buscou nas três últimas décadas renovar e transformar as práticas educativas no campo da saúde, ao incluir modelos de intervenção participativos, considerando o saber de todos os envolvidos. Assim, a partir desse modelo educativo-dialógico, que visa promover a transformação da realidade baseada na crítica e reflexão, as pessoas são estimuladas a tomar decisões sobre sua própria vida, por meio da noção de autonomia. Especula-se que quanto mais cedo forem proporcionadas atitudes de promoção de saúde, explicativas e ativas na disseminação do conhecimento acerca das condições de saúde, tais ações podem possibilitar uma mudança no cenário atual sobre saúde dos adolescentes, projetando adultos mais saudáveis ${ }^{6}$.

Diante da importância dessa temática, o presente artigo tem como objetivo relatar a experiência de uma ação de educação em saúde voltada para o público jovem, participantes de um curso preparatório para o Exame Nacional do Ensino Médio (ENEM).

MATERIAL E MÉTODO

Trata-se de um estudo descritivo, do tipo relato de experiência. Tal metodologia se caracteriza como de observação sistemática da realidade, sem a intenção de testar hipóteses, mas estabelecendo relações entre os achados da realidade com bases teóricas pertinentes ${ }^{7}$.

A obtenção dos resultados ocorreu por meio da descrição e reflexão acerca da experiência vivenciada no mês de setembro de 2019, em um curso preparatório para o Exame Nacional do Ensino Médio (ENEM), denominado Programa Complementar para Estudantes do Ensino Médio (PROCEEM), ofertado na Faculdade de Ciências da Saúde do Trairi (FACISA), unidade da Universidade Federal do Rio Grande do Norte (UFRN), na cidade de Santa Cruz, no estado do Rio Grande do Norte.

O PROCEEM é um programa desenvolvido em parceria entre a Pró-Reitoria de Graduação (PROGRAD) e a Pró-Reitoria de Extensão (PROEX), disponibilizado pela referida universidade. Consiste na oferta de aulas preparatórias gratuitas para alunos de escola pública que desejam ingressar no ensino superior e não possuem condições socioeconômicas de pagar por um serviço privado similar.

A experiência se deu por meio de ações construídas por dezesseis estudantes do curso de fisioterapia, do quarto, sexto e décimo períodos, participantes do projeto de extensão chamado "Educando em Saúde: conhecer para prevenir e promover saúde", o qual objetiva expandir o conhecimento à comunidade, educando em saúde sobre vários temas pertinentes a saúde de maneira geral, dentre eles, a saúde do homem, da mulher, e da pele.

O presente estudo segue em conformidade com as normas estabelecidas pela resolução no 510, de 07 de abril de 2016, do Conselho Nacional de Saúde, que orienta quanto às responsabilidades éticas de pesquisas nas ciências sociais e humanas. Além disso, não há exposição de dados que permitam a identificação do público alvo da ação, o que corrobora para a dispensa da apreciação por um Comitê de Ética e Pesquisa.

RELATO DA EXPERIÊNCIA

A ação de educação em saúde foi realizada pelos graduandos nas dependências da FACISA/UFRN, local de funcionamento das aulas do PROCEEM. Participaram da atividade 45 jovens, com idade entre 17 e 24 anos, de ambos os sexos.

A experiência iniciou-se uma semana antes da ação em si, com a capacitação dos estudantes de graduação que iriam realizar a ação de educação em saúde no curso preparatório sobre os temas que seriam abordados. Estes incluíam: prevenção do suicídio, conscientização da saúde do períneo, prevenção de doenças cardiovasculares, prevenção do câncer de intestino e a importância da fisioterapia neles. Os assuntos temáticos são discutidos e referentes ao mês de setembro, mês alusivo à conscientização/prevenção dos temas. E, as atividades planejadas são corpus para intervir em doenças de importância epidemiológica.

Diante disso, os graduandos se dividiram em grupos de três pessoas, em que cada um desses grupos ficaria com um assunto específico para abordagem. Os discentes do projeto de extensão pesquisaram a respeito da temática do seu grupo e preparam uma apresentação em formato de slides e 
metodologias ativas focadas nas rodas de conversa, com base nas evidências científicas atuais, compartilhando o conhecimento com os estudantes do ensino médio, como uma forma de estimulá-los a debater, o que permitiu que todos se apropriassem dos assuntos propostos.

A estratégia utilizada pelos participantes do projeto de extensão, enquanto graduandos, se caracterizou como educação permanente em saúde (EPS), que é compreendida como um conceito pedagógico que relaciona ensino, serviço, docência e saúde, contribuindo para o desenvolvimento profissional, a gestão setorial e o controle social. A EPS tem por base os pressupostos da aprendizagem significativa que devem ser orientadores das ações de desenvolvimento profissional e das estratégias de mudança das práticas de saúde ${ }^{8}$.

A metodologia utilizada para 0 compartilhamento do conhecimento foi a de sala de aula invertida, com abordagem teóricoprática, na qual os alunos respondiam a perguntas sobre assuntos de acordo com seus conhecimentos. Em seguida, os discentes participantes do projeto de extensão discutiam sobre a temática baseado no que foi abordado, como também mostravam a importância da fisioterapia nas respectivas condições. Para a fixação do conteúdo, os realizadores da ação optaram por um aplicativo online, de perguntas e respostas, denominado Kahoot, a fim de tornar ainda mais interativo e tecnológico. No entanto, nem todos possuíam celular com acesso à internet, de forma que foi necessária a divisão dos discentes do cursinho em grupos, a fim de que todos pudessem participar da dinâmica.

A princípio, os discentes de graduação falaram sobre o tema "conscientização da saúde do períneo". Para isso, dividiram a sala em 5 grupos, em que cada um retiraria uma pergunta e tentaria responder com suas próprias palavras, ou seja, não existiria resposta correta, uma vez que o objetivo era saber os conhecimentos dos participantes sobre 0 assunto. Cada grupo respondeu três perguntas sobre o tema. A partir do que os discentes do PROCEEM respondiam, os estudantes de fisioterapia discutiam junto com eles até chegar a uma explicação correta para a resposta da pergunta retirada. Em seguida, foi discutido sobre "prevenção ao suicídio", seguindo a mesma dinâmica do assunto anterior. Porém, no final da abordagem sobre suicídio, foi feito uma reflexão acerca da importância da vida e da existência de cada um. Posteriormente, os universitários discutiram ainda sobre "doenças cardiovasculares" e "câncer do intestino", respectivamente. Por fim, foi realizada a dinâmica com o uso do Kahoot.

O Kahoot é um aplicativo online em que as perguntas são repassadas para os participantes pelo moderador em uma tela. Para isso, cada pergunta será representada por um símbolo, por exemplo, quadrado, retângulo, triângulo, e só terá uma alternativa correta. Tais símbolos irão aparecer no celular dos participantes que acessarão um link específico do questionário para que eles marquem o que acharem que é correto. Logo, ganhava a equipe ou participante que respondesse corretamente 0 maior número de perguntas. Em caso de desempate, vence quem responder cada pergunta no menor intervalo de tempo.

DISCUSSÃO

A experiência relatada permite uma reflexão acerca da inserção da educação em saúde no ambiente educacional do ensino médio, principalmente para jovens, como meio de promover 0 empoderamento e conscientização.

Os espaços de ensino, como cursos preparatórios, são propícios para o desenvolvimento de ações de educação em saúde. A experiência vivenciada por cada jovem, associada ao compartilhamento dos temas abordados, pode contribuir para melhorar a percepção dos adolescentes sobre a saúde. Tradicionalmente, as escolas e o sistema de saúde abordam esses comportamentos por meio da educação em saúde ministrada nas salas de aula. Embora isso geralmente seja eficaz na divulgação do conhecimento e possa gerar mudanças de atitudes, a eficácia na redução de comportamentos de risco é irregular e geralmente de curto prazo ${ }^{9}$.

Outro aspecto relevante é que os estudantes que participaram dessa ação de extensão podem se tornar multiplicadores nas suas famílias, 0 que contribui para a disseminação de informação sobre temas relevantes para sociedade em que essas pessoas estão inseridas. Estudo demonstra que comportamentos de saúde são formados e os cursos de vida são traçados durante a segunda década de vida, a qual os participantes da ação estão inseridos. A educação para a vida familiar é o esforço pedagógico para enriquecer e melhorar a qualidade da vida dos indivíduos, como também a dos seus familiares, expandindo conhecimento e evitando problemas antes que eles ocorram ${ }^{10}$.

Concomitantemente a isso, o indivíduo jovem passa a ter mais autonomia no cuidado a sua própria saúde, a partir do que foi exposto na 
ação de educação desenvolvida em sala de aula. Nesse sentido, a vivência de experiências que demandam tomada de decisão de forma consciente, auxilia na constituição de autonomia. Para Paulo Freire, "é decidindo que se aprende a decidir", sendo que "faz parte do aprendizado da decisão a assunção das consequências do ato de decidir", e por isso é que "a decisão é um processo responsável". O mesmo também chama atenção para o fato de que a autonomia não acontece de repente, mas é um processo que precisa ser estimulado por experiências respeitosas, estimuladoras da decisão e da responsabilidade ${ }^{11}$.

Para obter benefícios de saúde a longo prazo, é de importância crucial que as mudanças nas atitudes resultem em decisões comportamentais autônomas. Tal ação é definida como o comportamento regulado pela escolha como expressão de si mesmo e geralmente se distingue do comportamento que é pressionado por forças intrapsíquicas e ambientais $^{12}$.

No que diz respeito aos discentes de fisioterapia que promoveram a ação, foi possível - compartilhamento do conhecimento dos assuntos abordados na graduação para pessoas da comunidade com linguagem clara e acessível, a fim de que fosse disseminado para - maior número de jovens, como também vivenciar a educação em saúde, que é um componente indispensável no processo de formação universitária.

As diretrizes curriculares dos Cursos de Graduação em saúde propõem que a formação do profissional de saúde deve contemplar o trabalho em equipe e a atenção integral à saúde. Assim, a atuação Fisioterapêutica não se restringe apenas ao campo curativo e da reabilitação, mas também nas ações de prevenção de doenças agravos e promoção da saúde, que envolvem a educação em saúde, como pilar da formação acadêmica ${ }^{13}$.

Nesse contexto, 0 graduando foi estimulado a entender ainda mais do processo de formação de outras pessoas e criar metodologias que envolvessem os alunos, como ocorreu na experiência, já que os participantes da ação se mostraram bem participativos e interagiram durante o decorrer das dinâmicas, tanto na sala de aula de invertida como no aplicativo online.

A partir disso, observou-se que os participantes tinham conhecimento dos assuntos discutidos, uma vez que responderam a maioria das questões de maneira assertiva, mesmo que em linguagem coloquial. Demonstraram empolgação quanto a discussão, pois expressaram acontecimentos do seu dia-adia e sanaram as possíveis dúvidas. Ou seja, colocaram-se como atores do processo de saúde-doença que havia sido exposto, com a possibilidade de se tornarem usuários empoderados do sistema de saúde. Além disso, eles se apresentaram comprometidos em reproduzir o conhecimento nas suas residências ou escolas, já que, segundo relatos, alguns dos exemplos dados pelos jovens faziam parte dos seus contextos sociais.

A sala de aula invertida considera que o conhecimento deve ser adquirido pelos alunos por meio de aprendizado individual antes da aula e a aplicação do conhecimento na resolução de problemas dentro do ambiente de aprendizado por meio de atividades centradas no aluno e facilitadas pelo docente ${ }^{14}$. Tal método de educação é chamado dialógico, em que o diálogo é essencial, já que o usuário é reconhecido como sujeito portador de um conhecimento, mas não é necessário que o saber do indivíduo seja condizente com o saber técnico-científico. Assim, o conhecimento prévio do sujeito nunca é deslegitimado, o que possibilita que a educação em saúde tenha, como ponto de partida, as condições concretas de vida das pessoas ${ }^{15}$.

Por sua vez, a tecnologia é uma grande aliada nas ações de produção da saúde. Ela pode ser classificada como educacional, assistencial e gerencial, indispensáveis na escolha dos dispositivos a serem utilizados nas ações. Os recursos promovem o conhecimento sobre as doenças, seus tratamentos, além suscitar o autocuidado ${ }^{16}$.

Outrossim, torna-se eficaz a humanização das atividades, em detrimento do contato direto com outros estudantes e a necessidade de adequar a linguagem científica, o que já capacita para possíveis atendimentos com a população.

No âmbito da saúde, a equipe na assistência deve utilizar uma linguagem acessível, capaz de realizar mediações entre diferentes públicos, que seja clara, objetiva, de fácil entendimento e principalmente que responda a expectativa do interlocutor ${ }^{17}$.

Até recentemente prevalecia o conceito de que "bastaria ser um excelente profissional, que consequentemente 0 indivíduo seria também um excepcional professor" nos planos de ensino dos cursos da área da saúde. Felizmente, o conceito está mudando e as oportunidades de desenvolvimento docente são cada vez mais ofertadas pelas instituições de ensino superior ${ }^{18}$, como no caso da experiência vivida. 
Paralelo ao ensino e a humanização, ainda existe o preparo para o mercado de trabalho, já que o fisioterapeuta se preocupa em suprir a demanda da comunidade com finalidade de reduzir os danos e agravos, executando uma prática integral que envolve educação em saúde, acolhimento, atendimentos individuais e em grupos, realizando visitas domiciliares, quebrando o paradigma de ser uma profissão apenas reabilitadora ${ }^{19}$. Características estas que foram exercitadas e desenvolvidas tanto na preparação como na experiência em si.

CONCLUSÃO

Destarte, fica evidente a importância da educação em saúde para jovens, especialmente no ambiente escolar, já que os participantes demonstraram empolgação quanto à discussão, expressando vivências do seu cotidiano e sanando as dúvidas que possuíam. Ou seja, colocaram-se como atores do processo de saúde-doença que havia sido exposto, com a possibilidade de se tornarem usuários empoderados do sistema de saúde. Além disso, eles se apresentaram comprometidos em reproduzir o conhecimento nas suas residências ou escolas, já que, segundo relatos, alguns dos exemplos dados pelos graduandos faziam parte dos seus contextos sociais. Por outro lado, a ação foi de suma importância também para os discentes de graduação, pois puderam vivenciar diversos eixos envolvidos na sua formação além do âmbito da universidade, como a educação em saúde, seja permanente ou continuada, humanização no atendimento, no caso da adequação da linguagem e contato direto com o público alvo, ensino, uma vez que se colocaram como educadores e criaram suas próprias metodologias, além do trabalho em equipe, em detrimento de toda a ação ter sido planejada e executada em grupo. Tal fato contribuiu diretamente para a formação de um profissional mais integral e humanizado.

\section{REFERÊNCIAS}

1. Malta DC, Neto OLM, Silva MMA, Rocha D, Castro AM, Reis AAC, Akerman M. National Health Promotion Policy (PNPS): chapters of a journey still under construction. Ciênc Saúde Coletiva. 2016;21(6):1683-94.

2. Malta DC, Reis AAC, Jaime PC, Neto OLM, Silva MMA, Akerman M. Ciênc Saúde Coletiva. 2018; 23(6):1799-809.

3. Gonçalves CB, Pinto ICM, França T, Teixeira $\mathrm{CM}$. The resumption of the implementation process of the National Permanent Health Education Policy in Brazil). Saúde Debate. 2019;43(esp.1):12-23.
4. Almeida ER, Moutinho CB, Leite MTS.Family health nurses' teaching practice in the health education development. Interface. 2016; 20(57):389-401.

5. Santili PGJ, Tonhom SFR, Marin MJS. Educação em saúde: algumas reflexões sobre sua implementação pelas equipes da Estratégia Saúde da Família. Rev Bras Promoç Saúde. 2016;29(Supl):102-10.

6. Viero VDSF, Farias JMD, Ferraz F, Simões PW, Martins JA, Ceretta LB. Educação em saúde com adolescentes: análise da aquisição de conhecimentos sobre temas de saúde. Esc Anna Nery. 2015;19(3):484-90.

7. Sousa BSA, Almeida CAPL, Carvalho HEF, Gonçalves LA, Cruz JN. A enfermagem no cuidado da criança autista no ambiente escolar. Saúde e Pesqui. 2018;11(1):163-70.

8. França $T$, Medeiros KR, Belisario SA, Garcia AC, Pinto ICM, Castro JL et al. Continuous Health Education policy in Brazil: the contribution of the Teaching-Service Integration Standing Committees. Ciênc Saúde Coletiva. 2017;22(6):1817-28.

9. Shackleton N, Jamal F, Viner RM, Dickson K, Patton G, Bonell C. School-Based Interventions Going Beyond Health Education to Promote Adolescent Health: Systematic Review of Reviews. J Adolesc Health. 2016;58(4):382-96.

10. Batra BS, Collins H. Effect of health education on adolescent girls' knowledge about family life education. Int $\mathrm{J}$ Health Care Edu Med Inform. 2019;6(1):3-5

11. Monteiro RJS, Oliveira MPCA, Belian RB, Lima LS, Santiago ME, Gontijo DT. DECIDIX: meeting of the Paulo Freire pedagogy with the serious games in the field of health education with adolescentes. Ciênc Saúde Coletiva. 2018;23(9):2951-962.

12. Koudenburg N, Jetten J, Dingle GA. Personal autonomy in group-basead interventions. Eur $\mathrm{J}$ Soc Psychol. 2017;47(5):653-60.

13. Alves NS, Portela ERM, Gonçalves FS, Guimarães TS, Alencar AJF, Mendes ES et al. Perspectivas sobre o trabalho do fisioterapeuta na atenção básica: uma revisão integrativa. Rev CPAQV. 2020;12(1):1-8.

14. Cotta RMM, Ferreira ES. Mapas conceituais e aula invertida: benefícios para o processo de ensino e aprendizagem sobre as políticas de saúde. RIEU. 2019;2(1):22-32.

15. Almeida ER, Moutinho CB, Leite MTS. Family health nurses' teaching practice in the health education development. Interface. 2016;20 (57):389-401.

16. Maniva SJCF, Carvalho ZMF, Gome RKG, Carvalho REFL, Ximenes LB, Freitas CHA. Educational technologies for health education on stroke: an integrative review. Rev Bras Enferm. 2018;71(4):1724-31. 
17. Assenheimer A, Moura D, Brum ZP, Fontana RT, Guimarães CA, Soares NV, Lucca JCP. Comunicação interpessoal enquanto fator para a humanização do cuidado. RICSB. 2018;2(2):1-9.

18. Bolleia VR, Cesaretti MLR. Sala de aula invertida na educação para as profissões de saúde: conceitos essenciais para a prática. Rev Eletr Farm. 2017;14(1):39-48.

19. Carvalho, VL, Oliveira, ALC. Interface entre a saúde coletiva e a fisioterapia: avaliação da política educacional. Fisioter Bras. 2016; 17(4):428-34

\section{CONFLITO DE INTERESSES}

Os autores declaram não haver conflitos de interesse

AUTOR PARA CORRESPONDÊNCIA

\section{João Pedro Santana Silva}

Avenida Rio Branco - Centro, ap 204

59200-000 Santa Cruz - RN, Brasil

E-mail: jp.santana428@gmail.com 